\title{
AN ASYMPTOTIC EXPANSION FOR A NONHOMOGENEOUS LINEAR SYSTEM ${ }^{1}$
}

\author{
THOMAS G. HALLAM
}

1. Introduction. An asymptotic expansion which is valid for all of the solutions of a nonhomogeneous linear system of differential equations of the form

$$
d y / d t=A(t) y+p(t)
$$

is developed in this article. In equation (1), $A(t)$ is an $n \times n$ matrix, $p(t)$ is an $n$-vector; both $A(t)$ and $p(t)$ have elements which are Lebesgue integrable on each finite subinterval of $[0, \infty)$. The fundamental matrix of the homogeneous equation associated with (1) which is equal to the $n \times n$ identity matrix at $t=t_{0}$ will be denoted by $\Phi\left(t ; t_{0}\right)$. The norm of a vector or a matrix is defined to be the sum of the absolute values of each of its components and will be designated by the symbol $\|\cdot\|$.

Results on the convergence of the solutions of a differential equation, by definition, yield asymptotic representations for the solutions. In [1], Bridgland demonstrates (under certain conditions) that the solutions $y(t)$ of (1) satisfy the equality

$$
\lim _{t \rightarrow \infty} y(t)=\left[\lim _{t \rightarrow \infty} \int_{t_{0}}^{t} \Phi(t ; \tau) d \tau\right]\left[\lim _{t \rightarrow \infty} p(t)\right] .
$$

A generalization of Bridgland's convergence was investigated in [3] by the author; in particular, for a given scalar function $\psi(t)$ an $n$-vector (matrix) $q(t)$ is called $\psi$-convergent as $t$ approaches infinity if there exists a constant $n$-vector (matrix) $L$ such that $q(t) \sim L \psi(t)$ as $t$ approaches infinity. Similarly, $q(t)$ is called $\psi$-bounded as $t$ approaches infinity if $q(t)=O(\psi(t))$ as $t$ approaches infinity. All order relations in this note are considered as " $t$ approaches infinity" and this qualifying phrase will usually not be stated. Some acquaintance with the content of reference [3] will be assumed since we will often refer to arguments and statements from that article.

We now state the sufficiency condition of Theorem 3 of [3] as

THEOREM 0 . Let $\phi, \psi$ be given scalar functions which are absolutely continuous on bounded subintervals of $[0, \infty)$ and satisfy the equality $\int^{\infty}\left|\phi(t) \psi^{-1}(t)\right| d t=\infty$. If

Received by the editors July 5, 1968.

1 This research was supported in part by the National Science Foundation under grants GP-7457 and GP-8921. 
(i) every solution of (1) is $\psi$-bounded for every $\phi$-bounded $p(t)$ [or, equivalently, $\left.\int_{l_{0}}^{t}\|\Phi(t ; \tau) \phi(\tau)\|=O(\psi(t))\right]$;

(ii) $\int_{z_{0}}^{t} \Phi(t, \tau) \phi(\tau) d \tau \sim \beta \psi(t)$ for some constant matrix $\beta$; then every solution $y(t)$ of (1) is $\psi$-convergent for every $\phi$-convergent $p(t)$.

The proof of the theorem reveals a representation similar to the above mentioned result of Bridgland. Namely, every solution $y(t)$ of (1) satisfies

$$
y(t) \sim \beta L \psi(t)
$$

where $L=\lim _{t \rightarrow \infty} \phi^{-1}(t) p(t)$; this result is indicated in equation (24) of [3]. Two questions arise concerning equation (2): First, (2) gives an asymptotic representation for the vector $y(t)$, can this be extended to an asymptotic expansion for the solutions $y(t)$ of (1)? Secondly, all of these solutions $y(t)$ are asymptotically equivalent at infinity, do they always agree in their asymptotic development?

By the choice of the functions $\phi$ and $\psi$ of the above theorem as $\phi(t)=t^{r}, \psi(t)=t^{m}$ with $r-m=q, q$ a nonnegative integer, and by assuming certain asymptotic power series expansions hold, we shall answer both questions affirmatively for these special cases.

2. The asymptotic expansion. We will denote by $Q_{k}(t), k \geqq 0$, the expression

$$
Q_{k}(t)=\int_{t_{0}}^{t} t^{-m+k} \Phi(t ; \tau) \tau^{r-k} d \tau
$$

and by $P_{k}(t)$

$$
P_{k}(t)=t^{m} Q_{k}(t) .
$$

From (4), it may be seen that $P_{k}(t)$ satisfies the nonhomogeneous matrix equation

$$
P_{k}^{\prime}=\left(A(t)+k t^{-1} I\right) P_{k}+t^{r} I
$$

where $I$ denotes the $n \times n$ identity matrix. Hence, another representation of $P_{k}(t)$ is $P_{k}(t)=\int_{t_{0}}^{t} \Psi(t ; \tau) \tau^{r} d \tau$ where $\Psi\left(t ; t_{0}\right)$ is the fundamental matrix of the system $z^{\prime}=\left(A(t)+k t^{-1} I\right) z$ satisfying $\Psi\left(t_{0} ; t_{0}\right)=I$. From the variation of parameters formula, we obtain an integral equation representation for $Q_{k}(t)$; namely,

$$
Q_{k}(t)=k \int_{t_{0}}^{t} t^{-m} \Phi(t ; \tau) \tau^{m-1} Q_{k}(\tau) d \tau+Q_{0}(t) .
$$

The next result is concerned with the asymptotic expansion of $Q_{k}(t)$. 
We shall adopt the summation convention that any sum of the form $\sum_{i=a}^{b}-$ whose terminal index $b$ is less than the initial index $a$-will be assumed to be zero; otherwise, the usual summation notation will be observed.

THEOREM 1. Let the hypothesis of Theorem 0 be satisfied wherein $\psi(t)=t^{m}, \phi(t)=t^{r}$ with $r-m=q, q$ a nonnegative integer. Furthermore, let $Q_{0}(t)$ possess the asymptotic power series expansion

$$
Q_{0}(t) \sim \sum_{i=0}^{\eta} C_{i, 0} t^{-i}, \quad 0 \leqq \eta \leqq \infty
$$

Then $Q_{k}(t)$ also has an asymptotic power series expansion given by

$$
Q_{k}(t) \sim \sum_{i=0}^{\eta} C_{i, 0} t^{-i}
$$

provided $q \geqq \eta$ and $k>0$.

If $q \leqq \eta-1$ and $k>0$, then $Q_{k}(t)$ has an asymptotic power series expansion given by

$$
Q_{k}(t) \sim \sum_{i=0}^{\eta} D_{i, k} t^{-i}
$$

where $D_{i, k}$ are recursively given as follows:

Suppose $C_{0, k}=C_{0,0}$ for all $k>0$, then $D_{i, k}$ is defined by

$$
\begin{aligned}
D_{i, k} & =C_{i, 0}, & i & =0,1, \cdots, q ; \\
& =C_{i, 0}+C_{i, k}, & i & =q+1, q+2, \cdots, \eta ;
\end{aligned}
$$

where

(9) $\quad C_{i, k}=k \sum_{j=0}^{i-q-1} D_{j, i-j} D_{i-q-1-j, k}, \quad i=q+1, q+2, \cdots, \eta$.

Proof. First, we shall show that the asymptotic expansion (7) holds when $q \geqq \eta$; to this end, an induction on the number of terms in the expansion of $Q_{k}(t)$, as given by $(7)$, will be used. Since $Q_{0}(t)$ possesses an asymptotic representation $Q_{0}(t) \sim C_{0,0}$ then the representation $Q_{k}(t) \sim C_{0,0}$ follows from equation (28) of [3]. Now, we shall assume that the expansion ( 7 ) is valid to $N-1$ terms, $N-1<\eta$; then, using the integral equation (5), we obtain 


$$
\begin{aligned}
& t^{N}\left[Q_{k}(t)-\sum_{i=0}^{N-1} C_{i, 0} t^{-i}\right] \\
& =t^{N}\left\{k \int_{t_{0}}^{t} t^{-m} \Phi(t ; \tau) \tau^{m-1}\left[Q_{k}(\tau)-\sum_{i=0}^{N-1} C_{i, 0} \tau^{-i}\right] d \tau\right. \\
& \left.\quad+k \sum_{i=0}^{N-1} \int_{t_{0}}^{t} t^{-m} \Phi(t ; \tau) \tau^{m-i-1} d \tau \cdot C_{i, 0}+Q_{0}(t)-\sum_{i=0}^{N-1} C_{i, 0} t^{-i}\right\} \\
& =k \int_{t_{0}}^{t} t^{N-m} \Phi(t, \tau) o\left(\tau^{r-q-N}\right) d \tau \\
& \quad+k \sum_{i=0}^{N-1} \int_{t_{0}}^{t} t^{N-m} \Phi(t ; \tau) \tau^{r-q-i-1} d \tau \cdot C_{i, 0}+C_{N, 0}+o(1) .
\end{aligned}
$$

Each of the quantities on the right side of the above equation will be considered separately. First, one can write

$$
\int_{t_{0}}^{t} t^{N-m} \Phi(t ; \tau) o\left(\tau^{r-q-N}\right) d \tau=\int_{t_{0}}^{t} t^{N-m} \Phi(t ; \tau) \tau^{r-N} \gamma(\tau) d \tau
$$

where $\gamma(t)=o(1)$. It will now be shown that there exists a positive constant $M$ such that

$$
\int_{t_{0}}^{t} t^{N-m}\|\Phi(t ; \tau)\| \tau^{r-N} d \tau \leqq M
$$

Using the definitions (3) and (4) along with the supplementary remarks following them we note that

$$
P_{N}(t)=\int_{t_{0}}^{t} \Psi(t ; \tau) \tau^{r} d \tau
$$

where $\Psi\left(t ; t_{0}\right)$ is the fundamental matrix of the equation

$$
z^{\prime}=\left(A(t)+N t^{-1} I\right) z
$$

such that $\Psi\left(t_{0} ; t_{0}\right)=I$. This differential equation may be regarded as a perturbation of the homogeneous equation $x^{\prime}=A(t) x$; hence, a calculation shows that $\Psi\left(t ; t_{0}\right)=t^{N} \Phi\left(t ; t_{0}\right) t_{0}{ }^{-N}$. Theorem 2 of [3], which is a result of perturbation type, used in conjunction with Theorem 1 of that paper implies that there exists a constant $M>0$ such that

$$
\int_{t_{0}}^{t} t^{-m+N}\|\Phi(t ; \tau)\| \tau^{r-N} d \tau=\int_{t_{0}}^{t} t^{-m}\|\Psi(t ; \tau)\| \tau^{\tau} d \tau \leqq M .
$$

Armed with this fact, the order relation 


$$
\int_{t_{0}}^{t} t^{N-m} \Phi(t ; \tau) o\left(\tau^{\tau-q-N}\right) d \tau=o(1)
$$

may be established as follows. For $\epsilon$ a given positive number, let $t_{1}>t_{0}$ be chosen so that $|\gamma(t)|<\epsilon / 2 M$ whenever $t \geqq t_{1}$. Select $t_{2} \geqq t_{1}$ sufficiently large such that $\int_{c_{0}}^{l_{1}} t^{N-m}\|\Phi(t ; \tau) \gamma(\tau)\| \tau^{r-N} d \tau<\epsilon / 2$ for $t \geqq t_{2}$; this is possible by virtue of Lemma 1 of [3]. Then, for $t \geqq t_{2}$,

$$
\begin{aligned}
\left\|\int_{t_{0}}^{t} t^{N-m} \Phi(t ; \tau) o\left(\tau^{r-q-N}\right) d \tau\right\| \leqq & \int_{t_{0}}^{t_{1}} t^{N-m}\|\Phi(t ; \tau) \gamma(\tau)\| \tau^{r-N} d \tau \\
& +\frac{\epsilon}{2 M} \int_{t_{1}}^{t} t^{N-m}\|\Phi(t ; \tau)\| \tau^{r-N} d \tau \\
& <\epsilon ;
\end{aligned}
$$

this verifies (11).

Also, as a consequence of the above proof, we have

$$
\int_{t_{0}}^{t} t^{N-m} \Phi(t ; \tau) \tau^{r-q-1-i} d \tau=\int_{t_{0}}^{t} t^{N-m} \Phi(t ; \tau) \tau^{r-N} o(1) d \tau=o(1) .
$$

The combination of the order relations (11) and (12) with equation (10) yields

$$
\lim _{t \rightarrow \infty} t^{N}\left[Q_{k}(t)-\sum_{i=0}^{N-1} C_{i, 0} t^{-i}\right]=C_{N, 0}
$$

which verifies $(7)$ in the case that $\eta \leqq q$.

Next, it will be shown that the expansion (8) holds for $\eta$ satisfying the inequality $q+1 \leqq \eta$. In this instance, certainly, the expansion $Q_{0}(t) \sim \sum_{i=0}^{q} C_{i, 0} t^{-i}$ is valid; hence, the previous case of the theorem can be applied to obtain the asymptotic expansion $Q_{k}(t) \sim \sum_{i=0}^{q} C_{i, 0} t^{-i}$.

Again the procedure used to establish (8) is induction; suppose that the expansion (8) is valid to $N-1$ terms, where $q \leqq N-1<\eta$; then, from (5), we obtain

$$
\begin{aligned}
t^{N}\left[Q_{k}(t)-\sum_{i=0}^{N-1} D_{i, k} t^{-i}\right]= & k \int_{t_{0}}^{t} t^{N-m} \Phi(t ; \tau) \tau^{m-1} o\left(\tau^{-N+1}\right) d \tau \\
& +\sum_{i=0}^{\eta} C_{i, 0} t^{N-i}+o\left(t^{N-\eta}\right)-\sum_{i=0}^{N-1} D_{i, k} t^{N-i} \\
& +k \sum_{i=0}^{N-1} \int_{t_{0}}^{t} t^{N-m} \Phi(t ; \tau) \tau^{m-1-i} d \tau \cdot D_{i, k}
\end{aligned}
$$


As noted above in the previous part of the theorem (see, e.g. (11)) we have

$$
\int_{t_{0}}^{t} t^{N-m} \Phi(t ; \tau) \tau^{m-N} o(1) d \tau=o(1) .
$$

The use of the definition of $D_{i, k}$ leads to the equality

$$
\begin{aligned}
& \sum_{i=0}^{\eta} C_{i, 0} t^{N-i}+o\left(t^{N-\eta}\right)-\sum_{i=0}^{N-1} D_{i, k} t^{N-i} \\
&=C_{N, 0}-\sum_{i=q+1}^{N-1} C_{i, k} t^{N-i}+o(1) .
\end{aligned}
$$

The last group of terms on the right side of equation (13) will now be considered; a rearrangement gives

$$
\begin{aligned}
k \sum_{i=0}^{N-1} \int_{t_{0}}^{t} t^{N-m} & \Phi(t ; \tau) \tau^{m-1-i} d \tau \cdot D_{i, k} \\
& =k \sum_{i=0}^{N-1} t^{N-q-1-i} \int_{t_{0}}^{t} t^{-m+q+1+i} \Phi(t ; \tau) \tau^{r-q-1-i} d \tau \cdot D_{i, k} .
\end{aligned}
$$

An application of the induction hypothesis in each of the special cases $k=i+q+1, i=0,1, \cdots, N-1$, yields the asymptotic expansion

$$
\int_{t_{0}}^{t} t^{-m+q+1+i} \Phi(t ; \tau) \tau^{r-q-1-i} d \tau \sim \sum_{j=0}^{N-1} D_{j, q+1+i} t^{-j},
$$

$i=0,1, \cdots, N-1$. Using this expansion and the definition of $C_{i, k}$ as given by (9) the right side of (16) may be written as

$$
\begin{aligned}
k \sum_{i=0}^{N-q-1} \sum_{j=0}^{N-q-1} D_{j, q+1+i} D_{i, k} t^{N-q-1-i-j}+o(1) \\
=k \sum_{i=0}^{N-q-1} \sum_{j=0}^{N-q-1-i} D_{j, N-i-j} D_{N-q-1-i-j, k} t^{i}+o(1) \\
=\sum_{i=0}^{N-q-1} C_{N-i, k} t^{i}+o(1) \\
=\sum_{i=q+1}^{N-1} C_{i, k} t^{N-i}+C_{N, k}+o(1) .
\end{aligned}
$$

The insertion of the conjunction of equations (16) and (17) together with the expressions (14) and (15) into equation (13) gives the de- 
sired expansion (8) when $q \leqq \eta-1$. This completes the proof of Theorem 1.

An asymptotic power series expansion for the solutions of (1) will now be demonstrated. For notational purposes we write

$$
Q_{k}(t) \sim \sum_{i=0}^{\eta} D_{i, k} t^{-i}
$$

where $D_{i, k}$ is given by the expression in the statement of Theorem 1 ; this will be done independent of the relative size of $q$ and $\eta$.

Theorem 2. Let the hypothesis of Theorem 1 be satisfied. If $Q_{0}(t)$ has the asymptotic expansion (6) and $p(t)$ has the asymptotic expansion

$$
p(t) \sim \sum_{i=0}^{\eta} p_{i} t^{r-i}
$$

then all solutions $y(t)$ of (1) possess the expansion

$$
y(t) \sim \sum_{i=0}^{\eta} \sum_{j=0}^{i} D_{i-j, j} p_{j} t^{m-i} .
$$

Proof. From the variation of parameters method we obtain

$$
t^{-m} y(t)=t^{-m} \Phi\left(t ; t_{0}\right) y_{0}+\int_{t_{0}}^{t} t^{-m} \Phi(t ; \tau) p(\tau) d \tau .
$$

For $\eta=0$ the conclusion of the above theorem reduces to that of Theorem 0 where the asymptotic representation is given by (2); proceeding inductively we now assume that the expansion (19) is valid for the integer $N-1<\eta$. From (20), it follows that

$$
\begin{aligned}
t^{N}\left[t^{-m} y(t)\right. & \left.-\sum_{i=0}^{N-1} \sum_{j=0}^{i} D_{i-j, j} p_{j} t^{-i}\right] \\
= & t^{N-m} \Phi\left(t ; t_{0}\right) y_{0}+\int_{t_{0}}^{t} t^{N-m} \Phi(t ; \tau) \tau^{r} e_{N+1}(\tau) d \tau \\
& +\sum_{i=0}^{N} \int_{t_{0}}^{t} t^{N-m} \Phi(t ; \tau) \tau^{r-i} d \tau \cdot p_{i}-\sum_{i=0}^{N-1} \sum_{j=0}^{i} D_{i-j, j} p_{j} t^{N-i}
\end{aligned}
$$

where

$$
e_{N+1}(t)=p(t) t^{-r}-\sum_{i=0}^{N} p_{i} t^{i}
$$

Lemma 1 of [3] yields the fact that $t^{N-m} \Phi\left(t ; t_{0}\right) y_{0}=o(1)$ and an 
argument like that quoted for (11) shows that

$$
\int_{t_{0}}^{t} t^{N-m} \Phi(t ; \tau) \tau^{r} e_{N+1}(\tau) d \tau=o(1),
$$

since $e_{N+1}(t)=o\left(t^{-N}\right)$. Thus, the right side of (21) becomes

$$
\begin{aligned}
\sum_{i=0}^{N} \int_{t_{0}}^{t} t^{N-m} \Phi(t ; \tau) \tau^{r-i} d \tau \cdot p_{i}-\sum_{i=0}^{N-1} \sum_{j=0}^{i} D_{i-j, j} p_{j} t^{N-i}+o(1) \\
=\sum_{i=0}^{N-1} t^{N-i}\left[\int_{t_{0}}^{t} t^{-m+i} \Phi(t ; \tau) \tau^{r-i} d \tau-\sum_{j=0}^{N-1-i} D_{j, i} t^{-j}\right] p_{i} \\
+\int_{t_{0}}^{t} t^{N-m} \Phi(t ; \tau) \tau^{r-N} d \tau \cdot p_{N}+o(1) .
\end{aligned}
$$

Theorem 1 may be applied to the quantity in the brackets above to yield

$$
\begin{array}{r}
\int_{t_{0}}^{t} t^{-m+i} \Phi(t ; \tau) \tau^{r-i} d \tau-\sum_{j=0}^{N-1-i} D_{j, i} t^{j}=D_{N-i, i} t^{(N-i)}+o\left(t^{-N+i}\right) \\
i=0,1, \cdots, N-1 .
\end{array}
$$

Inserting this result into equation (22) yields

$$
\begin{aligned}
t^{N}\left[t^{-m} y(t)-\sum_{i=0}^{N-1} \sum_{j=0}^{i}\right. & \left.D_{i-j, j} p_{j} t^{i}\right] \\
& =\sum_{i=0}^{N-1} D_{N-i, i} p_{i}+\int_{t_{0}}^{t} t^{N-m} \Phi(t ; \tau) \tau^{r-N} d \tau \cdot p_{N}+o(1) \\
& \sim \sum_{i=0}^{N} D_{N-i, i} p_{i} .
\end{aligned}
$$

This completes the proof of Theorem 2 .

REMARK 1. It is interesting to note that no additional hypotheses on our system are necessary (other than the expected ones of the existence of asymptotic expansions for $Q_{0}$ and $p$ ) to obtain an asymptotic expansion for the solutions rather than an asymptotic representation for the solutions. This does not happen for certain other sets of hypotheses; see e.g. Hille [4] for a linear second order scalar equation or [1], [5] for nonlinear extensions where additional (stronger) hypotheses are required to find higher order asymptotic expansions.

REMARK 2. The answer to the second question raised in the intro- 
duction concerning the total agreement of the asymptotic developments of the solutions of (1) is affirmative in the case considered here where an asymptotic power sequence is used (as indicated by Theorem 2). It is probable that in the general case all of the solutions have the same asymptotic expansion up to terms of order $t^{-m}\left\|\Phi\left(t ; t_{0}\right)\right\|$ which, as indicated by Lemma 1 of $[3]$, may be quite small.

\section{REFERENCES}

1. T. F. Bridgland, Jr., Asymptotic behavior of the solutions of nonhomogeneous differential equations, Proc. Amer. Math. Soc. 12 (1961), 546-552.

2. T. G. Hallam, Asymptotic expansions in a nonhomogeneous differential equation, Proc. Amer. Math. Soc. 18 (1967), 432-438.

3. - On the asymptotic growth of the solutions of a system of nonhomogeneous linear differential equations, J. Math. Anal. Appl. 25 (1969), 254-265.

4. E. Hille, Nonoscillation theorems, Trans. Amer. Math. Soc. 64 (1948), 234-252.

5. P. Waltman, On the asymptotic behavior of solutions of a nonlinear equation, Proc. Amer. Math. Soc. 15 (1964), 918-923.

Florida State University 